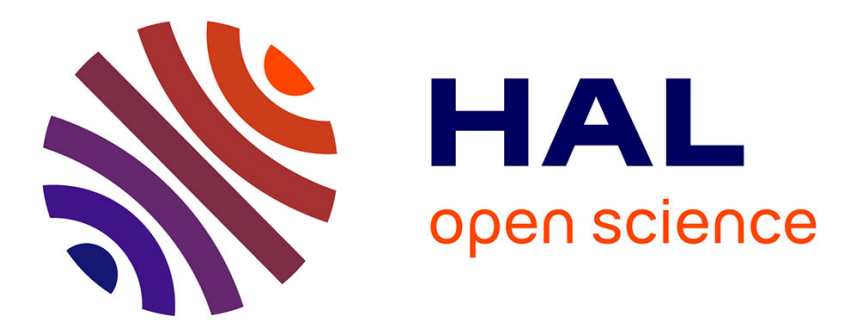

\title{
Photoacoustic study of collisional effects on rotational relaxation processes induced by infrared multiphoton absorption
}

\author{
M. Terzic, D. Markusev, J. Jovanovic-Kurepa
}

\section{- To cite this version:}

M. Terzic, D. Markusev, J. Jovanovic-Kurepa. Photoacoustic study of collisional effects on rotational relaxation processes induced by infrared multiphoton absorption. Journal de Physique IV Proceedings, 1994, 04 (C4), pp.C4-739-C4-742. 10.1051/jp4:19944203 . jpa-00252665

HAL Id: jpa-00252665

https://hal.science/jpa-00252665

Submitted on 1 Jan 1994

HAL is a multi-disciplinary open access archive for the deposit and dissemination of scientific research documents, whether they are published or not. The documents may come from teaching and research institutions in France or abroad, or from public or private research centers.
L'archive ouverte pluridisciplinaire $\mathbf{H A L}$, est destinée au dépôt et à la diffusion de documents scientifiques de niveau recherche, publiés ou non, émanant des établissements d'enseignement et de recherche français ou étrangers, des laboratoires publics ou privés. 


\title{
Photoacoustic study of collisional effects on rotational relaxation processes induced by infrared multiphoton absorption
}

\author{
M. TERZIC, D.D. MARKUSEV ${ }^{*}$ and J. JOVANOVIC-KUREPA* \\ University of Novi Sad, P.O. Box 224, 21001 Novi Sad, Serbia, Yugoslavia \\ * Institute of Physics, P.O. Box 57, 11001 Belgrade, Serbia, Yugoslavia
}

\begin{abstract}
The novel experimental approach is applied to investigate the cross sections for rotational relaxation $\sigma_{\text {rot.-rel. }}$ in the $\mathrm{SF}_{6}$-Ar mixtures irradiated by pulsed $\mathrm{CO}_{2}$ laser line. The applied method is the pulsed photoacoustic technique, and it is based on proposal for $\sigma_{\text {rot-rel. }}$ determination by transmission spectroscopy technique. The collisional effects have been investigated for the $0,1-0,8 \mathrm{~J} / \mathrm{cm}^{2}$ laser fluence range. Also, the collisional effects on rotational and translational temperature $\left(\mathrm{T}_{\mathrm{R}}\right)$ for $\mathrm{SF}_{6}-\mathrm{Ar}$ mixtures have been calculated.
\end{abstract}

\section{Introduction}

The phenomena known as the collision-induced enhancement of the multiphoton absorption (MPA) in $\mathrm{SF}_{6}$ molecules is suggested to be understood as the result of $\mathrm{SF}_{6}$ collisions with a nonabsorbing buffer-gas partners [1]. Actually, it is proposed that the dramatic increase in the absorption cross sections for $\mathrm{SF}_{6}$ irradiated by $\mathrm{CO}_{2}$ laser light, is due to it's rotational relaxation into states depleted by laser pumping. Now is accepted that collisions play a variety of roles in MPA processes, but rotational relaxation could be very important phenomena, while couples the states that are not being pumped by the laser, to those that are [2]. So called, "rotational hole filling" effect actually follows the collision-induced rotational relaxation by absorbing and also nonabsorbing partners in investigated gas mixtures.

The method suggested for $\sigma_{\text {rot-rel. }}$ measurements [3] is based on the transinission spectroscopy (TRS) technique. It incorporates two assumptions: a) the rotational relaxation time of the absorbing molecule is the most important parameter which determines the laser pulse transmission trough the gas, and b) the appropriate analytical representation of the transmission process is one given for the steady-state response of a saturable absorber [4].

For a better understanding of the rotational relaxation phenomena, we have used the photoacoustic method (PAS) instead of proposed TRS method. The reason for this is the much higher sensitivity of PAS technique in comparison to TRS method.

Our experiment was performed in the gas mixtures of $\mathrm{SF}_{6}$ molecules $\left(\mathrm{p}_{\mathrm{SF} 6}=0,47\right.$ mbar $)$ and $\mathrm{Ar}$ (in the pressure range 1-140 mbar) as the buffer-gas. The mixtures were irradiated by the pulsed $\mathrm{CO}_{2}$ laser (FWHM $45 \mathrm{~ns}$ ) operated at the 10P(16) line, in the fluence range $0,1-0,8$ $\mathrm{J} / \mathrm{cm}^{2}$. Althought the TRS and PAS approaches are similar, the results obtained for $\sigma_{\text {rot-rel. }}$ by PAS technique seem to be more precise.

\section{Experimental Technique and Data Reduction}

The details about the experimental apparatus used to obtain the necessary data for $\sigma_{\text {rot-rel. }}$ determination, are given somewherels [5]. This setup is actually the high sensitivity PAS method, in which the normalization to absolute scale is performed by TRS devices used 
simultaneously with the photoacoustic detector. The block diagram of the apparatus and some basic information are given in [5].

For the illuminated gas in which rotational relaxation dominates, what has been found for gas mixtures (similar to our): by extensive numerical computation [6], the, so called, saturation intensity $I_{s}$ (photons $/ \mathrm{cm}^{2}$ ) is defined [4] as

$$
\mathrm{I}_{\mathrm{s}}=\mathrm{n}<\mathrm{v}>\sigma_{\text {rot }- \text { rel. }} / \sigma_{a}(\phi)
$$

where $\mathrm{n}$ is the density of the molecules, $<\mathrm{v}>$ their mean relative velocity, $\sigma_{a}$ is the molecular absorption cross section for specific laser line at the fluence $\phi\left(\mathrm{J} / \mathrm{cm}^{2}\right)$. On the other hand, for an optically thick absorber $I_{s}$ is also defined [4] as

$$
I_{s}=\frac{4}{h v d^{2} t_{i}} \cdot \frac{E_{i n}-E_{t}}{\ln \frac{E_{t}}{E_{i}}+\alpha_{0} p L}
$$

where $h v$ is the $\mathrm{CO}_{2}$ photon energy, $\mathrm{d}$ is the radius of the laser beam, $\mathrm{t}_{\mathrm{i}}$ is the laser pulse duration time, $\mathrm{E}_{\text {in }}$ and $\mathrm{E}_{\mathrm{t}}$ are incoming and transmitted laser excitation energy through the cell of the length $\mathrm{L}$, and $\alpha_{0}$ is absorption coefficient of the gas mixture at the pressure $\mathrm{p}$.

Let us define the absorbed laser energy along the length of the photoacoustic cell $(\mathrm{L})$ as $E_{a b}$. Then $E_{a b}=E_{i n}-E_{t}=S(p, \gamma, T) P_{a}$, where $S(p, \gamma, T)$ is already determined sensitivity of the used photoacoustic gas cell-detector system. $P_{a}(m V)$ is the relevant photoacoustic signal measured at gas mixture pressure $p$ and for incoming laser light intensity $E_{i}$. When $S(p, \gamma, T)$ and $P_{a}$, as the basic parameter, are measured, $I_{s}$ could be also expressed in terms of this quantities, i.e.,

$$
I_{s}=\frac{4}{h v d^{2} \pi t_{i}} \cdot \frac{S(p, \gamma, T) P_{a}}{\ln \left[1-\frac{S(p, \gamma, T) P_{a}}{E_{i}}\right]+\alpha_{0} p l_{m}}
$$

where $1_{m}$ being the length of the used photoacoustic microphone. From eq. (1) and eq. (3) $\sigma_{\text {rot.-rel. }}$ is obtained for the absorbing gas molecules:

$$
\sigma_{\text {rot-rel. }}(p, \phi)=\frac{4}{h v d^{2} \pi t_{i}} \cdot \frac{1}{\langle v\rangle} \cdot \frac{l_{m} E_{i} \sigma_{a}^{2}(\phi)}{\ln \left[1-n l_{m} \sigma_{a}(\phi)\right]+\alpha_{0} p l_{m}}
$$

It is rather simple to obtain, in the case of the gas mixtures, where with $A$ is the molecular absorber of the light designated, and with $\mathrm{B}$ the nonabsorbing collisional partner, the following relation:

$$
\sigma_{\text {rot. }- \text { rel. }}^{B}=\frac{\sigma_{a}(\phi)}{n_{B}<v_{B}>} \cdot\left[I_{s}^{B}-I_{s}^{A}\right]
$$

where $\sigma_{\text {rot.-rel. }}^{B}$ defines the rotational relaxation cross section of the absorbing particle $A$ under the collision of nonabsorbing $B$ species.

\section{Experimental Results and Discussion}

For the $\mathrm{SF}_{6}-\mathrm{Ar}$ mixtures the systematic measurements have been performed, to obtain $\sigma_{\text {rot.-rel. }}$ using PAS technique and $\mathrm{SF}_{6}$ gas at the constant pressure. All photoacoustic measurements have been taken for already calibrated photoacoustic system, i.e. for every Ar pressure the apparatus sensitivity $S(p, g, T)$ was known. Than, the research program has been 
incorporated the measurements for the three different laser light fluences $(0.49,0.30$ and 0.19 $\left.\mathrm{J} / \mathrm{cm}^{2}\right)$, for which the relevant photoacoustic data $\left(P_{a}\right.$ and $\left.E_{i}\right)$ have been registrated by $P C$ computer, as well as them for every Ar pressure in the investigated pressure range (1-140 mbar).

In Fig. 1a the changes of $\sigma_{\text {rot.-rel. }}$ are shown as the function of Ar gas pressures which

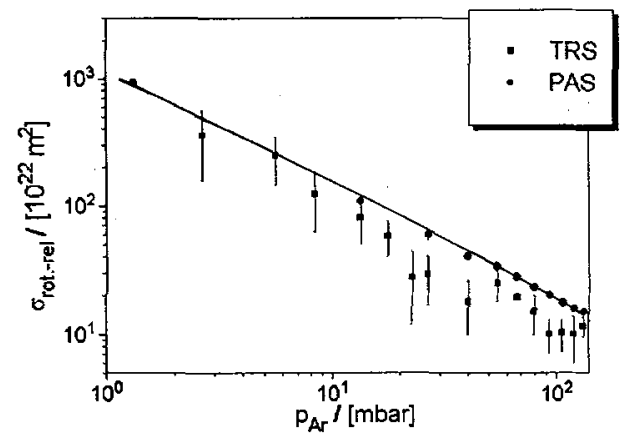

Figure 1.a. $\sigma_{\text {rol } \text { rd }}$ dependence of Ar pressure and comparison of the results from TRS and PAS measurements in our case.

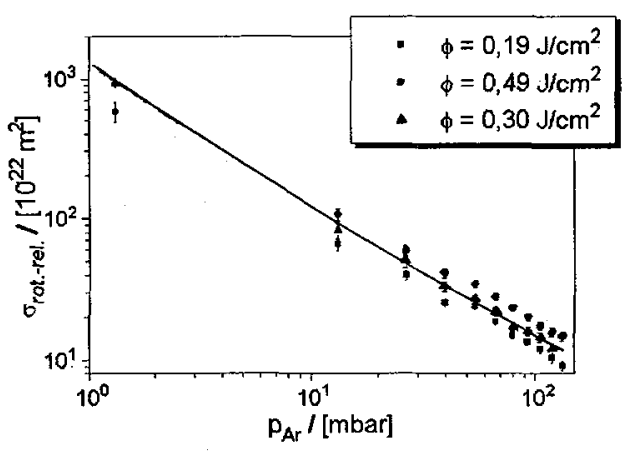

Figure 1.b. $\sigma_{\text {mol-red }}$ dependence of Ar pressure for different incoming laser fluences obtained in our experiments.

is the characteristic of $\mathrm{SF}_{6}$ molecules diluted by Ar. In our apparatus $\sigma_{\text {rot.-rel. }}$ could be obtained by two experimental methods (PAS and TRS) independently or simultaneously. In Fig. 1a two independent measurements have been used to demonstrate the quality of the data obtained by PAS approach. First one, at $\phi=0,494 \mathrm{~J} / \mathrm{cm}^{2}$, is taken by TRS method and second, at nearly identical fluence $\phi=0,486 \mathrm{~J} / \mathrm{cm}^{2}$, is taken by PAS method. The quality of the results obtained by PAS due to much higher sensitivity is obvious, in comparison with the results obtained by TRS technique.

In Fig. $1 \mathrm{~b} \sigma_{\text {rot.-rel. }}$ for $\mathrm{SF}_{6}$ are shown for three different laser fluences obtained by PAS method. It is evident the dramatic decrease, of nearly three orders of magnitude for $\sigma_{\text {rot.-rel. }}$ values, as a function of Ar gas pressure, as well as, but not so dramatic one for smaller laser fluences.

In Fig. $2 \mathrm{a} \sigma_{\text {rot-rel. }}$ for $\mathrm{SF}_{6}$ under the collision of $\mathrm{SF}_{6}$ molecules (eq. 4) are given as the function of the laser fluence. Just the opposite behavior for $\sigma_{\text {rot.-rel. }}$ has been established in this case than in the case presented in Fig. $1 b$.

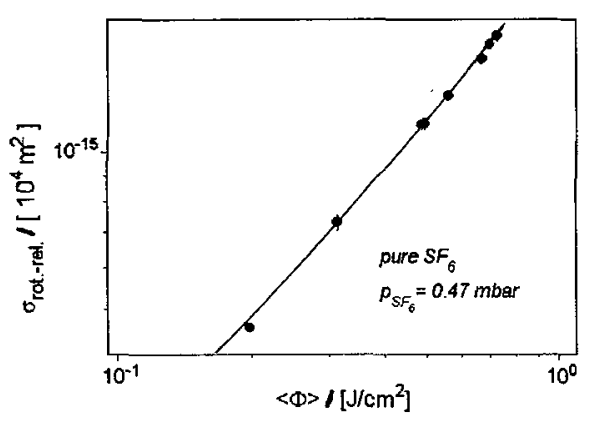

Figure 2.a. $\sigma_{\text {rat-red }}$ values dependence of laser incoming fluence for pure $\mathrm{SF}_{6}$ in the gas-cell system.

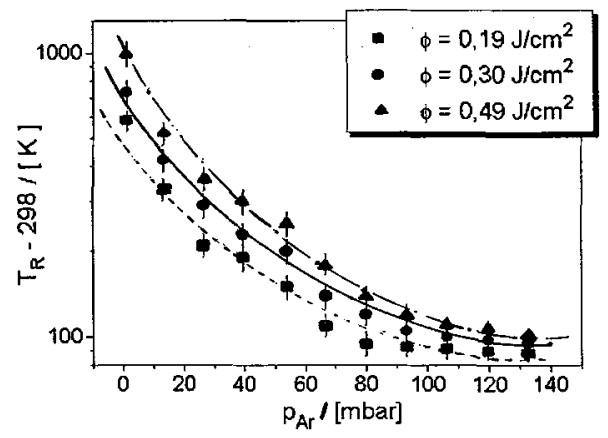

Figure 2.b. $T_{R}$ values dependence of Ar pressure for different laser incoming fluences. 
However, it was of interest to determine, so called, $T_{R}$ quantity, with the aim of better understanding of MPA process in $\mathrm{SF}_{6}-\mathrm{Ar}$ mixtures. $\mathrm{T}_{\mathrm{R}}$ (in $\mathrm{K}$ ) is the rotational translational temperature of the gas mixture. It is defined [7] as

$$
T_{R}-298=\frac{2<n>h v}{3 R} \cdot \frac{p_{S F_{6}}}{p_{\text {buffer-gas }}}
$$

where $\langle\mathrm{n}\rangle$ is the average number of the absorbed photon per one molecule $\left(\mathrm{SF}_{6}\right)$, usually calculated from $\langle n\rangle=\sigma_{0} \phi / h v, \mathrm{R}$ is the Rydberg constant, while $h v$ is the laser photon energy. In Fig. $2 b \mathrm{~T}_{\mathrm{R}}$ is given for $\mathrm{SF}_{6}-\mathrm{Ar}$ mixtures as the function of $\mathrm{Ar}$ gas pressure. The results are also presented for the different laser fluences. Values for $T_{R}$ calculated from eq. (6) represent the "adiabatic" temperatures, i.e. temperatures reached by the unexpanded irradiated volume.

\section{Conclusions}

In this contribution, the use of the PAS technique is demonstrated for $\sigma_{\text {rot.-rel. }}$ measurements as well as its superiority in comparison with TRS technique (Fig. 1a). The common opinion that the rotational relaxation processes could be the very important processes $[1,2]$ for "rotational hole filling" mechanism, it looks, is valid only for the low pressure regime $\left(p_{A r} \leq 10\right.$ mbar). The dramatic decrease of $\sigma_{\text {rot.-rel. }}$ and $\mathrm{T}_{\mathrm{R}}$ values for the buffer gas (Ar) pressure $p>10$ mbar (Fig. lb and Fig. 2b) are not doubtful. This facts, in our opinion, are indication for substantial increase in the probabilities for $\mathrm{V}-\mathrm{V}$ and $\mathrm{V}-\mathrm{T}$ energy transfer processes at pressures $\mathrm{p}_{\mathrm{Ar}}>10$ mbar. Investigation of $\mathrm{V}-\mathrm{T}$ relaxation in $\mathrm{SF}_{6}-\mathrm{Ar}$ mixtures are in progress.

\section{References}

[1] P. Q. Quigley, Opt. Lett., 3, (1978), 106

[2] "Multiple-Photon Excitation and Dissociation of Polyatomic Molecules", Ed. by C. D. Cantrell, Springer-Verlag, (1986), 63

[3] F. M. Lussier, J. I. Steinfeld, T. F. Deutsch, Chem. Phys. Lett., 58, (1978), 277

[4] M. Hersher, Appl. Opt., 6, (1967), 947

[5] J. Jovanovic-Kurepa, M. Terzic, D. D. Markusev and P. Vujkovic Cvijin, submitted to Measur. Sci. and Techn.

[6] D. G. Satton, I. Burak and J. I. Steinfeld, IEEE J. Quantum Electron., QE-7, (1971), 82

[7] M. Lenci, E. Molinari, G. Piciacchia, V. Sessa and M. L. Terranova, Chem. Phys, 142, (1990), 473 